Statistics Corner

Questions and answers about language testing statistics:

\title{
Standard error vs. Standard error of measurement
}

\author{
James Dean Brown (University of Hawai'i at Manoa)
}

QUESTION: One of the statistics my spreadsheet gives me is Standard Error. Is that the same as the Standard Error of Measurement?

ANSWER: The most direct answer to your question is "no." Most likely, you are referring to the STEYX function in the ubiquitous Excel ${ }^{\mathrm{TM}}$ spreadsheet. The statistic calculated by the STEYX function is commonly referred to as the standard error of estimate and that is not the standard error of measurement. As your question suggests, the standard error of estimate is often confused with the standard error of measurement that is reported by some test analysis software, or even with the standard error of the mean that is reported by more sophisticated statistical packages like SPSS, SAS, or SYSTAT. Let me try to unscramble all of this step by step by first reviewing what the standard deviation is. Then I will be able to explain the definitions and differences among the standard error of the mean, the standard error of estimate, and the standard error of measurement.

\section{Standard Deviation}

As I defined it in Brown (1988, p. 69), the standard deviation "provides a sort of average of the differences of all scores from the mean." This means that it is a measure of the dispersion of scores around the mean. The standard deviation is related to the range (another indicator of dispersion based on the distance between the highest and lowest score), but has the advantage over the range of not being affected as much as the range by aberrant scores that are exceptionally high or low. Generally, a low standard deviation means that a set of scores is not very widely dispersed around the mean, while a high standard deviation indicates that the scores are more widely dispersed. [For more information on calculating and interpreting standard deviations see Brown, 1988 and 1995]

It turns out that, in a normal distribution, about $68 \%$ of the students can be expected to fall in the range of scores between minus one standard deviation below the mean and plus one standard deviation above the mean and that about $95 \%$ of the students can be expected to fall in the range of scores between minus two standard deviations below the mean and plus two standard deviations above the mean. So on a test with a mean of 51 and standard deviation of 10 , you can expect about $68 \%$ of the students to score between 41 and 61 , and about $95 \%$ of the students to score between 31 and 71. This use of percents with the standard deviation will become important in interpreting all three of the standard error statistics described below. Now, having reviewed the basic concept of standard deviation, it is possible to consider the concept of standard error of the mean. 
However, if you were to select random samples of 50 students one after the other until you had 100 such random samples and administered the same test to all the students, you would find that the 100 samples would have many different means. Moreover, if you were to plot the means for the 100 random samples you would find that a histogram of those means would probably be normal in distribution and that the means themselves would have a mean (this mean of means, by the way, would probably be the best estimate of the population mean). The standard deviation of such a distribution of means is referred to as the standard error of the mean because it represents the distribution of errors (or random fluctuations) in estimating the population mean. Thus the standard error of the mean is the standard deviation for the distribution of errors or random fluctuations that are likely to occur in estimating the population mean from sample means in a particular situation. Based on the percentages discussed in the previous section for the standard deviation, we can expect about $68 \%$ of the errors to be distributed within one standard error plus or minus of the population mean, and about $95 \%$ to be distributed within two standard errors plus or minus of the population mean. Normally, you will not have the time or resources to actually take 100 samples. Instead, you may want to use the following formula to estimate the standard error of the mean from a single sample:

$$
S_{M}=\frac{S}{\sqrt{N}}
$$

Where:

$$
\begin{aligned}
& S_{M}=\text { standard error of the mean } \\
& S=\text { standard deviation of the mean } \\
& N=\text { number of scores on the test }
\end{aligned}
$$

So, if you had 50 students $(N)$ on a test with a standard deviation $(S)$ of 4.89 , the standard error of the mean $(S M)$ would turn out to be as follows:

$$
\begin{aligned}
S_{\text {MII }} & =\frac{S}{\sqrt{N}}=\frac{4.89}{\sqrt{50}}=\frac{4.89}{7.07} \\
& =6.917 \approx 6.9
\end{aligned}
$$

[For further explanation of standard error of the mean, see Hatch \& Lazaraton, 1991]

\section{Standard Error of Measurement}

Conceptually, the standard error of measurement is related to test reliability in that it provides an indication of the dispersion of the measurement errors when you are trying to estimate students' true scores from their observed test scores. In order to understand the previous sentence you will first need to understand three bits of jargon: sampling errors, true scores, and test scores. I will deal with them in reverse order. 
Students' test scores are not a mystery: they are simply the observed scores that the students got on the whatever test is being considered. However, that same students' true scores are a bit more difficult to understand. Let's say that you can give a test an infinite number of times to a group of students (I know fatigue would probably become a problem sometime before infinity, but this is just for the sake of argument). If you could average the students' scores over the infinite number of administrations, the average of each person's scores would probably be the best estimate of that person's true ability/knowledge in whatever is being tested, or that person's true score. The standard deviation of all those scores averaged across persons and test administrations is the standard error of measurement.

Of course it is humanly impossible to administer a test an infinite number of times while holding testing effect, fatigue, and other variables constant. So we settle for second best. We assume that each student's test score is our best estimate of the true score, but we recognize that there are sampling errors in that estimate, just as there were for estimating the population mean. Those sampling errors are normally distributed and have a standard deviation called the standard error of measurement.

Fortunately, an estimate of the standard error of measurement can be calculated from the test score standard deviation and reliability estimate using the following formula:

$$
S E A=\mathrm{S} \sqrt{1-r_{\mathrm{K}}}
$$

\section{Where: $\quad S E M=$ standard error of measurement$$
S=\text { standard deviation of the test }
$$$$
r_{x x}=\text { reliability of the test }
$$

So, if you have a test with a standard deviation $(S)$ of 4.89 , and a Cronbach alpha reliability estimate $\left(r_{x x}\right)$ of .91 , the standard error of measurement would be calculated as follows:

$$
\begin{aligned}
\mathcal{S E M}= & \mathrm{S} \sqrt{1-\mathrm{r}_{\mathrm{X}}}=4.89 \sqrt{1-.91}=4.89 \sqrt{.09} \\
& 4.89(.3)=1.467 \approx 1.47
\end{aligned}
$$

One useful application of the standard error of measurement is that it can be used to estimate a band of scores around any cut-point wherein students are treated with special care. For instance, if the test in question had a cut-point for failing of 30 , you should recognize that, if you want to be $68 \%$ sure of your decision, the standard error of measurement indicates that the students within one SEM of the cut point (i.e., $30+-1.47$, or 28.53 to 31.47 ) might fluctuate randomly to the other side of the cut point if they were to take the test again, so it might behoove you to gather additional information (e.g., other test scores, homework grades, interviews with the students, or whatever is appropriate) in deciding whether or not they should pass the test. 
By extension, if you want to be $95 \%$ sure of your decision, the standard error of measurement indicates that the students within two SEMs of the cut point (i.e., $30+-2.94$, or 27.06 to 32.94 ) might randomly fluctuate to the other side of the cut point, and you may want to act accordingly. [For further explanation of the standard error of measurement, see Brown, 1996, or Brown with Wada, 1999].

\section{Standard Error of Estimate}

Conceptually, the standard error of estimate is related to regression analysis in that it typically provides an estimate of the dispersion of the prediction errors when you are trying to predict $Y$ values from $X$ values in a regression analysis. In order to understand the previous sentence, you will first need to understand three bits of jargon: prediction errors, $Y$ values, and $X$ values. Again, I would like to deal with those terms in reverse order. In a regression analysis, $X$ values are any values from which you want to predict, and $Y$ values are any values to which you want to predict. Unfortunately, those predictions are never perfect because prediction errors occur. Such errors may be due to unreliable measurement in either the $Y$ or $X$ variable, or due to unsystematic differences between the two sets of numbers. When you are trying to predict $Y$ values from $X$ values, it would be useful to know what the distribution of those prediction errors is so you can interpret your predictions wisely.

An example of such a situation might be a case where you use regression analysis to predict TOEFL scores from the PERFECT test at your institution. You must first conduct a study based on a large number of students who took both tests. Then using regression analysis, you build a regression equation of the form $Y=a+b X$. Based on your analysis, you will know the values of $a$ (the intercept) and $b$ (the slope), and can then plug in the $X$ value (or PERFECT test score) for a student who has never taken the TOEFL. Solving for $Y$ will then give you that student's predicted $Y$ (or predicted TOEFL score). All of this is beyond the scope of this explanation, but is necessary in order to get even a basic understanding of what the standard error of estimate is. [For more on regression analysis, see Brown, 1988, or Hatch and Lazaraton, 1991].

We assume that any student's predicted $Y$ score is our best estimate of that score, but we recognize that there are sampling errors around that estimate, just as there were for estimating the population mean and true scores. Those sampling errors are normally distributed and, in this case, have a standard deviation called the standard error of estimate.

Fortunately, you can use the following simple formula to calculate the standard error of estimate from the standard deviation of the $Y$ values in the original regression analysis and the correlation coefficient between the $X$ and $Y$ values in that analysis:

$$
\text { see }=S_{Y^{\prime}} \sqrt{1-r_{Y_{X}}}
$$


Where:

$S E E=$ standard error of estimate

$S_{Y}=$ standard deviation of the $Y$ values in the original regression analysis

$r_{Y X^{2}}=$ correlation squared of $Y$ and $X$ values in the original regression analysis

So, if you have a set of $Y$ values (say test scores) with a standard deviation (SY) of 10.21 and a correlation with the $X$ values of .84 , the standard error of estimate would be calculated as follows:

$$
\begin{aligned}
& \text { see }=S_{Y} \sqrt{1-r_{Y X}}=10.21 \sqrt{1-.84}=10.21 \sqrt{.16} \text {. } \\
& 10.21(.4)=4.084 \approx 4.08
\end{aligned}
$$

One useful application of the standard error of estimate is that it can be used to create a band of scores around any predicted score. For instance, if a students predicted $Y$ score is 35, the see (just calculated) of 4.08 would tell you that the student's predicted score is likely to vary within plus or minus 4.08 points on $Y 68 \%$ of the time. So you know that the prediction would fall between 30.92 and 39.08 with $68 \%$ confidence. At this point, you can probably guess how you would go about establishing bands of scores for $95 \%$ confidence. Such bands of confidence intervals around predictions are very useful in making decisions based on predictions. [For further explanation of the standard error of estimate, see Brown, 1988 or Hatch and Lazaraton, 1991].

\section{Conclusion}

Quite obviously, the standard deviation, standard error of the mean, standard error of measurement, and standard error of estimate are quite different things. They are all based on the simple notions of the normal distribution, but they have quite different applications. In a nutshell:

1. The standard deviation helps you estimate the dispersion in a given distribution;

2. The standard error of the mean helps you to estimate the dispersion of sampling errors when you are trying to estimate the population mean from a sample mean;

3. The standard error of measurement helps you estimate the dispersion of the measurement errors when you are making decisions about students' scores at a certain cut-point; and

4. The standard error of estimate helps you estimate the dispersion of prediction errors when you are trying to predict $Y$ values from $X$ values in a regression analysis.

They are all useful statistical tools, and now you are in a position not only to understand the differences and similarities among these four concepts, but also to know when and where to apply them. 


\section{References}

Brown, J. D. (1988). Understanding research in second language learning: A teacher's guide to statistics and research design. London: Cambridge University Press.

Brown, J. D. (1996). Testing in language programs. Upper Saddle River, NJ: Prentice Hall.

Brown, J. D. (trans. by M. Wada). (1999). Gendo kyoiku to tesutingu. [Language teaching and testing]. Tokyo: Taishukan Shoten.

Hatch, E., \& Lazaraton, A. (1991). The research manual: Design and statistics for applied linguistics. Rowley, MA: Newbury House.

HTML: http://www.jalt.org/test/bro_4.htm ～/ PDF: http://www.jalt.org/test/PDF/Brown4.pdf 